\title{
Crystallization Behavior of Copper Tetra-tert-Butyl-Porphyrazine in Ultrathin Films
}

\author{
N. Borovkov, A. Kolker
}

G.A. Krestov Institute of Solution Chemistry, Russian Academy of Sciences

Address: 1, Akademicheskaya St., 153045 Ivanovo, Russia

E-mail: phthalocyanine2020@gmail.com

\section{Content}

\begin{tabular}{ccc}
\hline Part 1 & Preparation of CuPaz & Page S2 \\
\hline Part 2 & General Structural Data & Page S3 \\
\hline Part 3 & Layers on the Water Surface & Page S5 \\
\hline Part 4 & Morphology of the LS Films & Page S7 \\
\hline Part 5 & Structure of the LS Films & Page S8 \\
\hline Part 6 & Optical Spectra of All Films & Page S9 \\
\hline
\end{tabular}




\section{Part 1. Preparation of CuPaz}

All compounds and supplementary data in Part 1 is a private gift of Professor Vadim N. Kopranenkov (NIOPIK, Moscow) to the corresponding author.

An as-synthesized sample of $\mathrm{H}_{2} \mathrm{Paz}$ consists of three geometric isomers (Figure S1) to be separated by preparative column chromatography. The low-symmetric isomers are non-planar and, hence, bound to silica through $\mathrm{H}$ bonding. In contrast, the $\mathrm{C}_{4 \mathrm{~h}}-\mathrm{H}_{2} \mathrm{Paz}$ isomer is eluted without retention. Its purity is confirmed by NMR spectroscopy (Figure S2).

In this work, an as-synthesized sample was divided into two fractions: the neat $\mathrm{C}_{4 \mathrm{~h}}-\mathrm{H}_{2} \mathrm{Paz}$ isomer and a mixture of two others. The fractions were converted into copper derivatives $(\mathrm{Cu}-$ Paz) by reaction with copper acetate in pyridine. Also a mixture of three isomers (mix-CuPaz) was prepared.

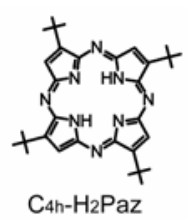

$58.4 \%$

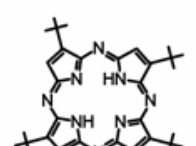

$\mathrm{C}_{\mathrm{s}}-\mathrm{H}_{2} \mathrm{Paz}$ $25.2 \%$

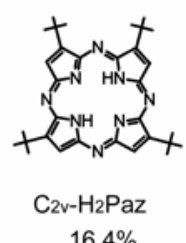

$16.4 \%$
Figure S1. Geometric isomers of freebase tetra-tert-butyl-porphyrazine.

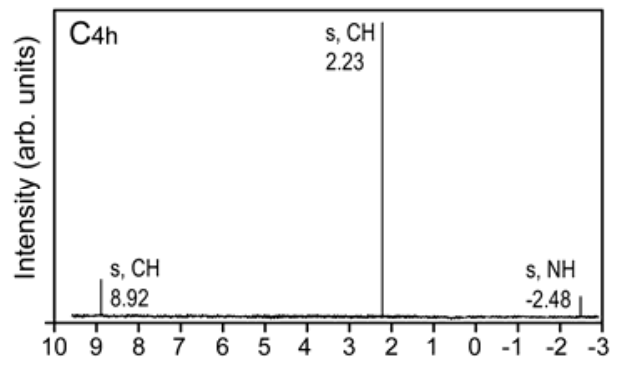

Figure S2. The ${ }^{1} \mathrm{H}$ NMR spectrum of $\mathrm{C}_{4 h}-\mathrm{H}_{2} \mathrm{Paz}$. Conditions: Bruker WM$360(360 \mathrm{MHz}), 1 \mathrm{mM}$ solution in $\mathrm{CDCl}_{3}$, room temperature, TMS standard. 


\section{Part 2. General Structural Data}

A drop-cast film of mix-CuPaz was prepared by placing a $5 \mu \mathrm{l}$ droplet of the ca. $2 \mathrm{mM}$ solution onto quartz glass cleaned with hydrogen peroxide. Upon solvent evaporation, the film split into orbicular islands (Figure S3). Then the film was annealed on a thermo-stable of a Boetius microscope.

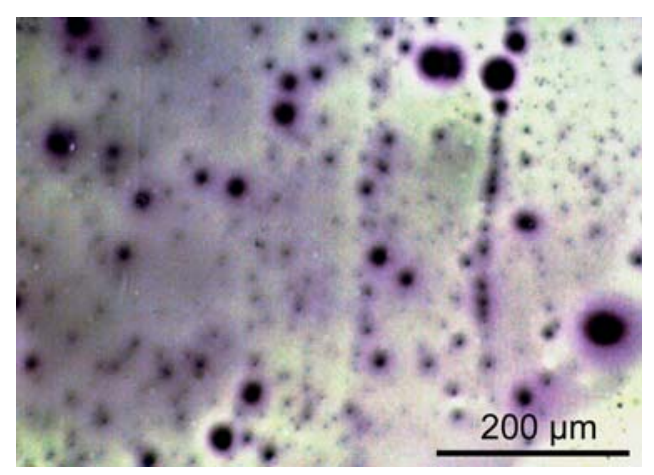

Figure S3. A light microscopy image of a mix-CuPaz film drop-cast from benzene solution. A slightly covered location is demonstrated.

Figure S4 shows an initial crystallization stage where rosette-like bundles of prismatic and flaky particles have arisen from the amorphous dye matter.

The thin-film crystallization of mix-CuPaz into prismatic particles was performed at $120-150{ }^{\circ} \mathrm{C}$. Relevant structural data are presented in Figures S5, S6, and Table S1.

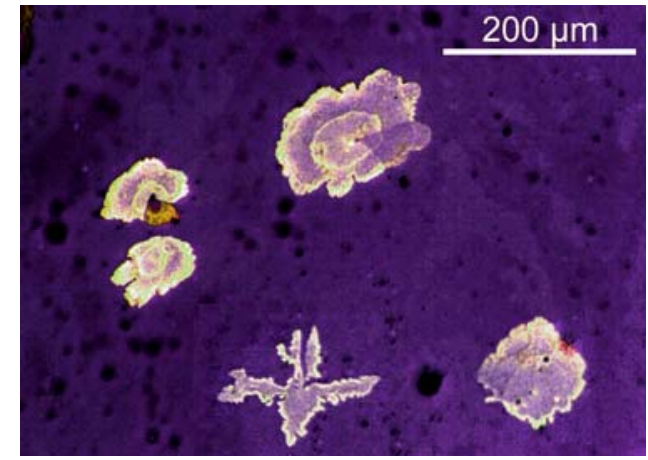

Figure S4. A light microscopy image of a mix-CuPaz film (Figure S3) annealed at $100{ }^{\circ} \mathrm{C}$. A heavily covered location is demonstrated.

The wide-angle X-ray scattering (WAXS) study was conducted on a Bruker D8 Advance apparatus using the $\mathrm{Ni}$-filtered $\mathrm{Cu}-\mathrm{K \alpha}$ radiation $(\lambda=$ $0.15418 \mathrm{~nm}$ ) with incident angles $(2 \Theta)$ varying from $2^{\circ}$ to $40^{\circ}$ and a step size of $0.02^{\circ}$.

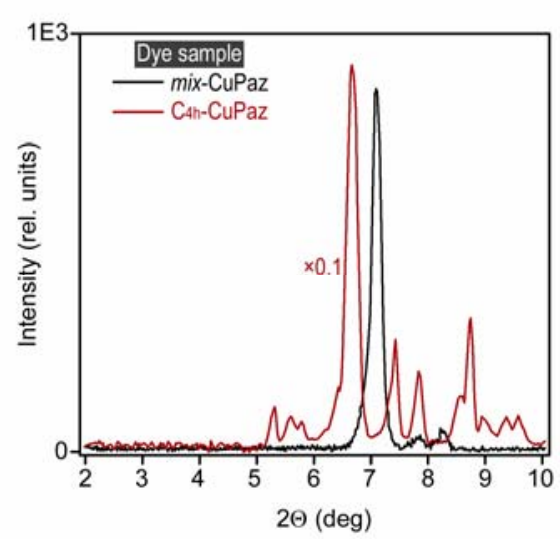

Figure S5. Diffraction patterns of dye crystallites: black, mix-CuPaz annealed at $150{ }^{\circ} \mathrm{C}$; red, $\mathrm{C}_{4 \mathrm{~h}}-\mathrm{CuPaz}$ as obtained from NIOPIK, Moscow. 


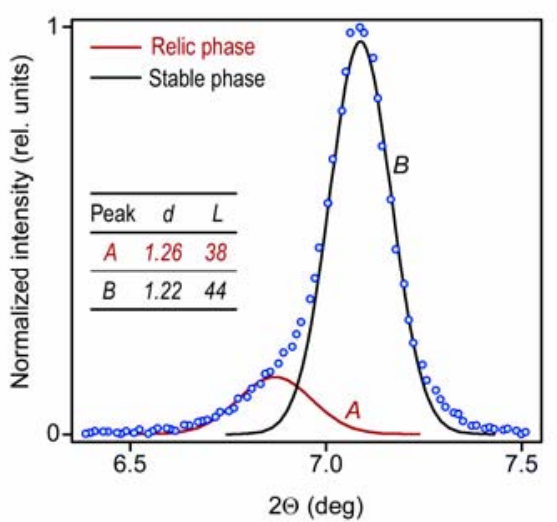

Figure S6. WAXS data for prismatic crystallites of mix-CuPaz (points) and fitting thereof by using the Gaussian function. The inserted table displays structural parameters of the solid phases $(\mathrm{nm}): d$, lattice periodicity; $L$, correlation length.

Table S1. WAXS data for $\mathrm{C}_{4 \mathrm{~h}}-\mathrm{CuPaz}$ (the red curve in Figure S5).

\begin{tabular}{ccccc}
\hline \hline Peak & $\mathbf{2 \Theta}$ & $\boldsymbol{d}$ & $\boldsymbol{L}$ & $\boldsymbol{I}$ \\
\hline 1 & 5.28 & 1.67 & 44 & 18 \\
\hline 2 & 5.59 & 1.58 & 27 & 16 \\
\hline 3 & 5.78 & 1.53 & 50 & 15 \\
\hline 4 & 6.66 & 1.33 & 22 & 100 \\
\hline 5 & 7.40 & 1.19 & 37 & 33 \\
\hline 6 & 7.82 & 1.13 & 34 & 25 \\
\hline 7 & 8.55 & 1.03 & 28 & 24 \\
\hline 8 & 8.73 & 1.01 & 49 & 37 \\
\hline 9 & 8.96 & 0.98 & 31 & 13 \\
\hline 10 & 9.34 & 0.95 & 27 & 12 \\
\hline 11 & 9.57 & 0.92 & 34 & 11 \\
\hline \hline
\end{tabular}

Abbreviations: $\Theta, d$, and $L$ as in

Figure S6; I, relative intensity, \%.
To keep the X-ray beam properly focused, the Bragg-Brentano parafocusing geometry was applied. The scattering intensity was measured by a scintillation counter with count time of 4 sec per step.

Figure S6 was specially worked out to demonstrate the existence of a crucially important phase termed "relic" here and "transient" in the main text. 


\section{Part 3. Layers on the Water Surface}

To extract a structural information from a compression $(\Pi-A)$ curve, one should draw a corresponding $\Pi A-\Pi$ plot, find inflection points, and associate these points with pressure-induced events on the water surface.

Figure S7 presents four truncated compression curves and a full curve specially recorded for analysis. Our analytical approach is explicated in Figures S8-S10.

The ПА-П plot (Figure S9, insert) has two linear fragments corresponding to phase-homogeneous dye layers. The slope of such fragments is equal to the molecular area $\left(A_{\mathrm{mol}}\right)$, while the $\Pi A$ quantity has a physical meaning of the layer compression work.

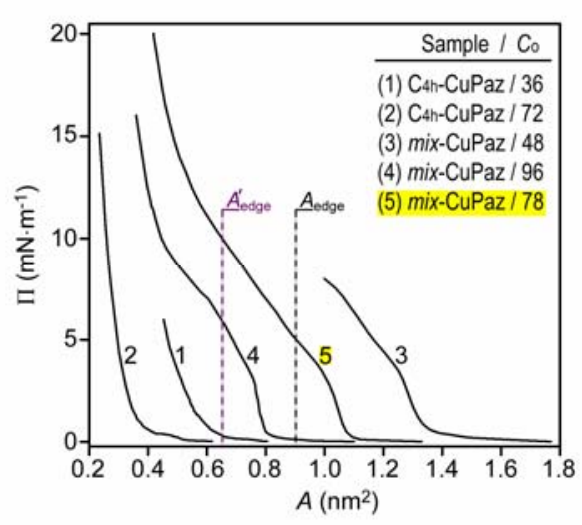

Figure S7. Compression curves for the floating $\mathrm{CuPaz}$ layers at different initial surface coverage $\left(C_{0}, \%\right)$. The fully recorded curve 5 is highlighted.

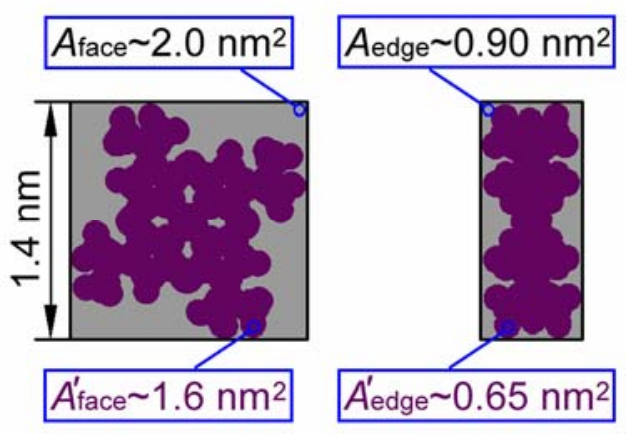

Figure S8. Calculated limiting areas that can be occupied by a single $\mathrm{C}_{4 \mathrm{~h}^{-}}$ $\mathrm{CuPaz}$ molecule on the water surface.

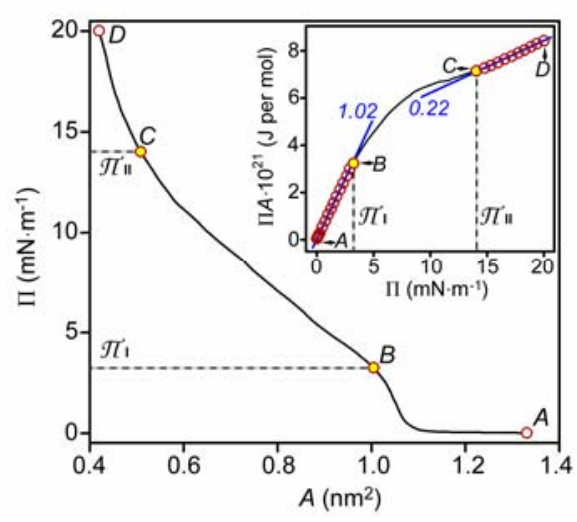

Figure S9. The curve 5 (highlighted in Figure S7) with inflection points $(B$ and $C$ ) extracted from the $\Pi A-\Pi$ plot (insert). Data points belonging to linear fragments of the $\Pi A-\Pi$ plots are depicted as red circles. The $\mathscr{T}_{\mathrm{I}}$ and $\mathscr{T}_{\mathrm{II}}$ symbols mark order/disorder boundaries. Blue italic decimals show molecular areas $\left(A_{\mathrm{mol}}, \mathrm{nm}^{2}\right)$.

As a first step, consider the curve 5 in Figure S9. The $\Pi A-\Pi$ plot has a short linear fragment $A-B$ ranging from 0 to ca. 3.5 (i.e., $\pi_{\mathrm{I}}$ ) $\mathrm{mN} \mathrm{m}^{-1}$. The slope of this fragment is equal to (1.02 
$\pm 0.01) \mathrm{nm}^{2}$, indicating the monolayer with tilted molecular arrangement. The next fragment $B-C$ corresponds to the disordered layer undergoing a phase transformation. Upon ultimate compression, there arises a polylayer with $A_{\text {mol }}$ of $(0.22 \pm 0.01) \mathrm{nm}^{2}$ being close to a quarter of the $A_{\text {edge }}$ value (Figure S8). Thus, one observes the direct monolayer $\rightarrow$ tetralayer phase transformation going on until $\Pi$ of $14.0 \mathrm{mN}$ $\mathrm{m}^{-1}$ (i.e., $\mathcal{T}_{\text {II }}$ ) is achieved.

Figure S10 allows understanding what layers have been used for the film deposition.

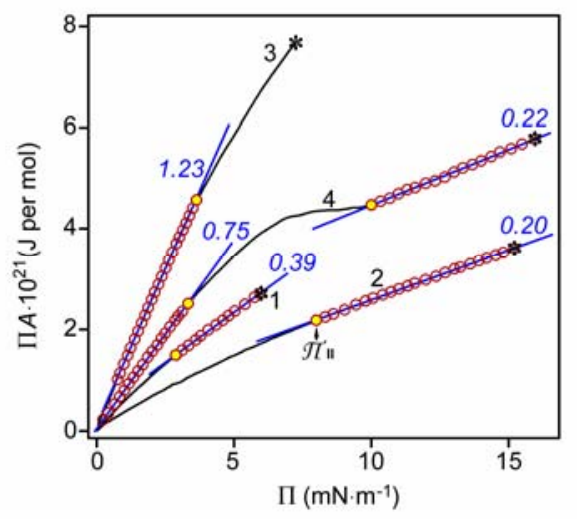

Figure S10. ПА-П plots for the curves 1-4 (Figure S7). All symbols are as in Figure S9. Transfer pressures are indicated by asterisks; $\mathscr{T}_{\mathrm{II}}=8.0 \mathrm{mN} \mathrm{m}^{-1}$.

Notes: 1) Under usual experimental conditions, $\mathrm{C}_{4 \mathrm{~h}}-\mathrm{CuPaz}$ forms only polymolecular layers (i.e., non-Langmuir bi- and tetra-layers). 2) In contrast, mix-CuPaz forms a variety of monolayers, in which the molecular tilting progressively increases with increasing $C_{0} .3$ ) In both cases, the most stable low-dimensional colloidal phase is represented by the tetralayer, in which the dye molecules are arranged vertically. 
Part 4. Morphology of the LS Films

Well covered locations are presented in the main text; Figure S11 shows morphological defects.

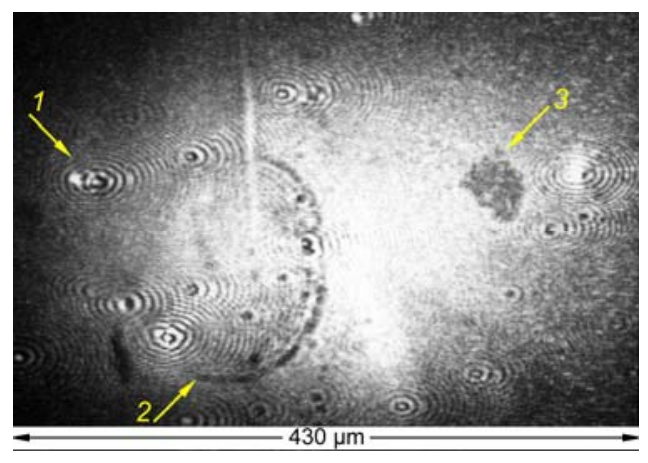

Figure S11. A BAM image of the three-layer LS film of mix-CuPaz deposited on a silicon wafer at $\Pi$ of 16 $\mathrm{mN} \mathrm{m}^{-1}$ and aged during 6 months. Morphological defects: (1) orbicular islands surrounded with concentric circles; (2) an arc-shaped groove; (3) a randomly dewetted site.
Crystalline LS films of $\mathrm{C}_{4 \mathrm{~h}}-\mathrm{CuPaz}$ were visualized by scanning electron microscopy (SEM). Figure $\mathbf{S 1 2}$ shows the film built from badly agglomerated lamellar particles.

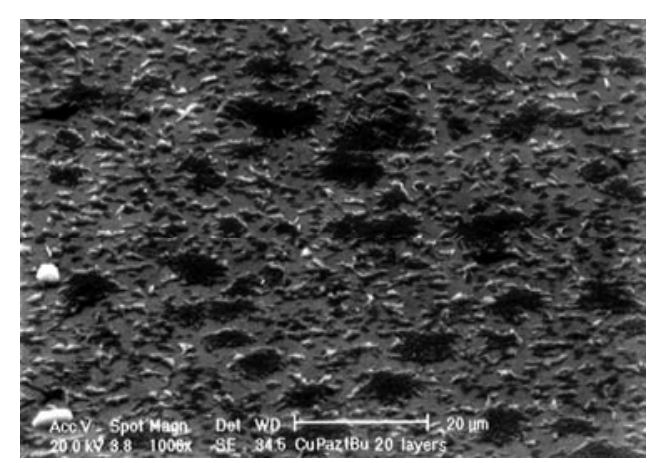

Figure S12. A SEM image of the $\mathrm{C}_{4 \mathrm{~h}^{-}}$ CuPaz film prepared by 20 -fold dipping of a silicon wafer into the floating layer at $6 \mathrm{mN} \mathrm{m}^{-1}$ and aged during 1 month. 


\section{Part 4. Structure of the LS Films}

Small-angle X-ray scattering (SAXS) study was conducted on the AMUR-K diffractometer (Figure S13) constructed under the auspices of Professor D. I. Svergun in the Special Designing Bureau of the L. V. Shubnikov Institute of Crystallography, Moscow (https://www.crys.ras.ru/tsc). All diffraction patterns were acquired using the $\mathrm{Ni}$-filtered $\mathrm{Cu}-\mathrm{K} \alpha$ radiation.

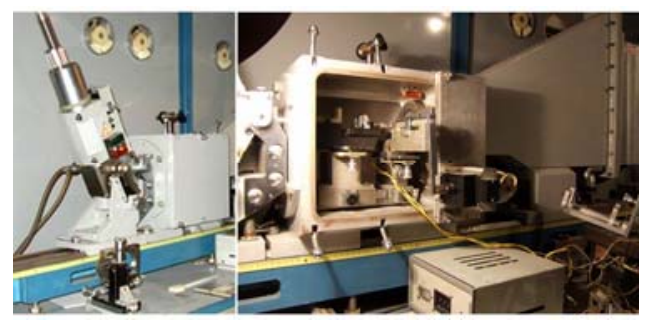

Figure S13. Small-angle AMUR-K diffractometer.

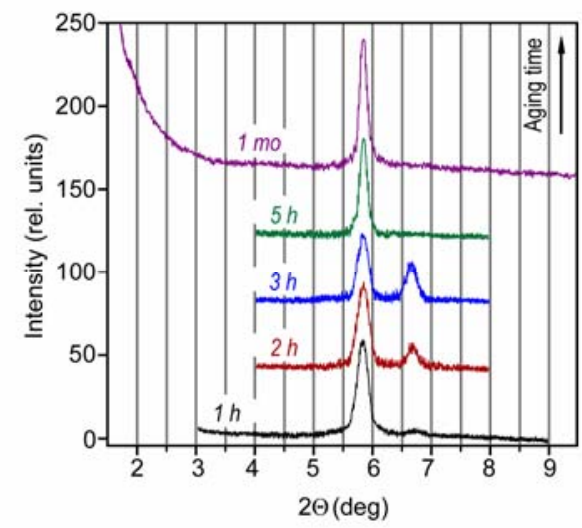

Figure S14. Diffraction patterns of the $\mathrm{C}_{4 \mathrm{~h}}-\mathrm{CuPaz}$ film deposited at $\Pi$ of $6 \mathrm{mN}$ $\mathrm{m}^{-1}$. Aging times are inscribed. The $1^{\text {st }}$ trace is shown as recorded; the others are offset by $40 n$ units $(n=1-4)$.

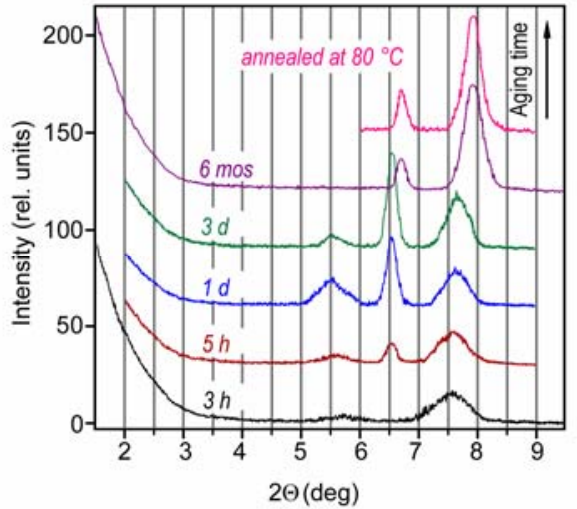

Figure S15. Diffraction patterns of the mix-CuPaz film deposited at $\Pi$ of 8 $\mathrm{mN} \mathrm{m}{ }^{-1}$. Aging times are inscribed. The $1^{\text {st }}$ trace is shown as recorded; the others are offset by $30 n$ units $(n=1-$ $5)$.

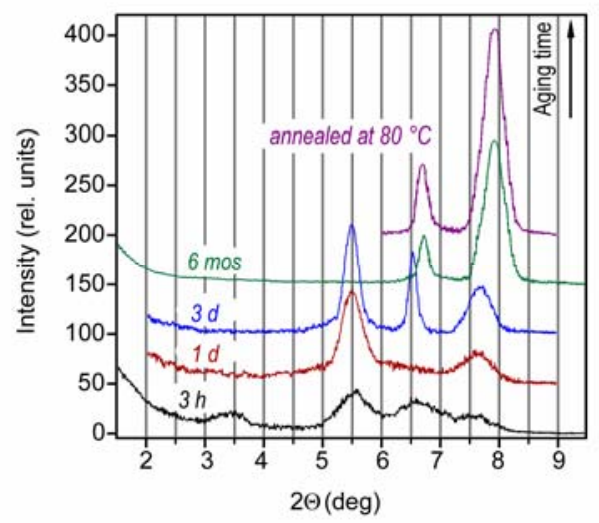

Figure S16. Diffraction patterns of the mix-CuPaz film deposited at $\Pi$ of 16 $\mathrm{mN} \mathrm{m}{ }^{-1}$. Aging times are inscribed. The $1^{\text {st }}$ trace is shown as recorded; the others are offset by $50 n$ units $(n=1-$ 4). 
Part 5. Optical Spectra of All Films

Key experimental data and relevant analytics are included in Section $\boldsymbol{A}$. Section $\boldsymbol{B}$ contains auxiliary data.

In a standard experiment, a dye film was annealed in two steps (gently at $80 \pm 1{ }^{\circ} \mathrm{C}$ and severely at $150 \pm 2$ ${ }^{\circ} \mathrm{C}$ ) by using a thermo-table of a Boetius microscope. Annealing time was $1 \mathrm{~h}$. No decomposition and sublimation of the dye occurred under these conditions.

In the case of $\mathrm{C}_{4 \mathrm{~h}}-\mathrm{CuPaz}$, a dropcast film was deposited from solution in benzene added with a small amount of freshly distilled 3-dimethylamino-1propanol (DMAP) from SigmaAldrich.

To obtain reproducible data, a holder with a square window $(7 \times 7$ $\mathrm{mm}$ ) was made of a thick brass foil. A film-covered glass slide was placed over the window and attached with two brass micro-clips. When measuring a film spectrum, the holder was placed in a fixed position into a sample compartment of Specord M400.

\section{Section $A . \mathrm{C}_{4 \mathrm{~h}}-\mathrm{CuPaz}$ films.}

Note: Figures S17, S18, and S19 are related to the LS film of $\mathrm{C}_{4 \mathrm{~h}^{-}}$ CuPaz, whereas Figures S20, S21, and
Table S2 show data on the drop-cast film.

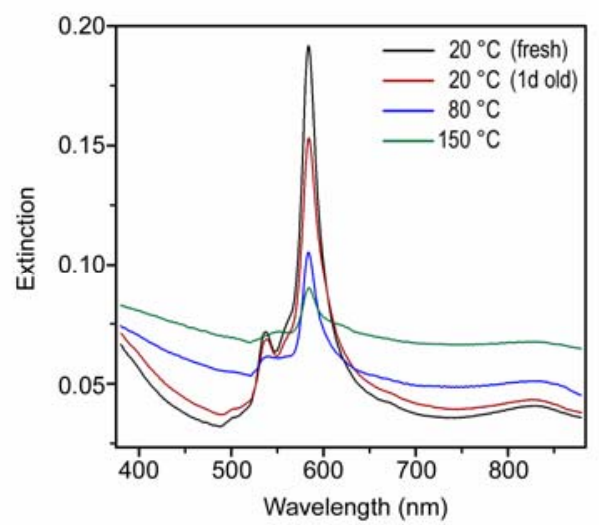

Figure S17. Optical spectra of the LS film of $\mathrm{C}_{4 \mathrm{~h}}-\mathrm{CuPaz}$ (20 layers on quartz). Annealing time is $1 \mathrm{~h}$.

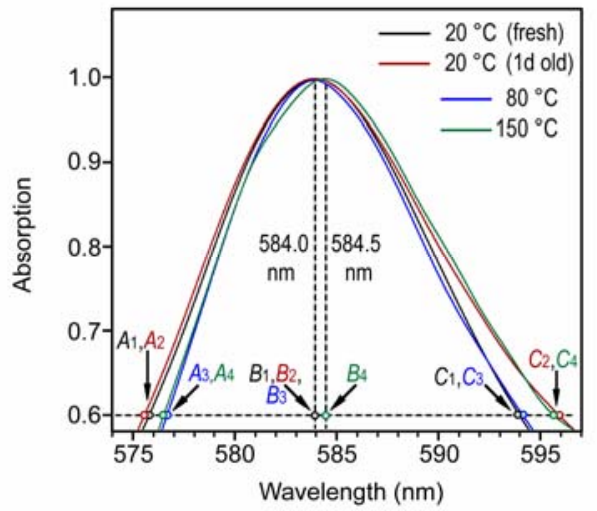

Figure S18. The $Q$ band profiles (Figure S17) analyzed on a subject of peak asymmetry estimated as a $B C / A B$ ratio ( $\pm 2.5 \%$ ): 1.25 (black); 1.41 (red); 1.33 (blue); 1.38 (green).

Note: The $Q$ band profile changes irregularly upon heat treatment, shifting to the red in a stepwise manner. 

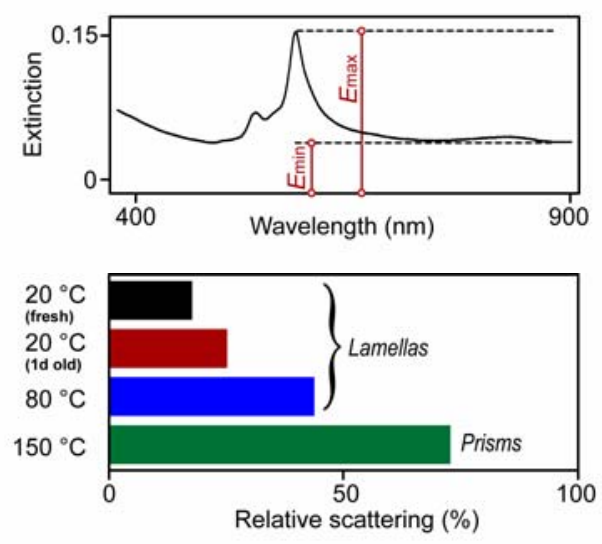

Figure S19. Optical spectra of the LS film (Figure S17) analyzed on a subject of scattering intensity. The relative scattering quantity is estimated as $100 \times\left(E_{\min } / E_{\max }\right)$.

Note: This quantity for the film annealed at $150{ }^{\circ} \mathrm{C}$ is overestimated because of solid-state dewetting that accompanies the lamella-to-prism transformation.

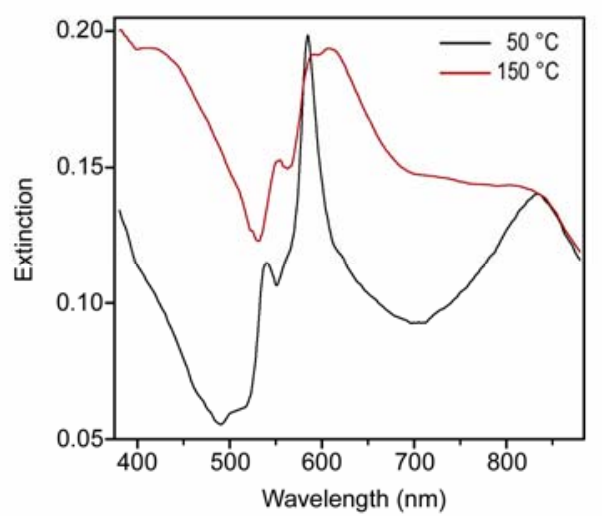

Figure S20. Optical spectra of the $\mathrm{C}_{4 \mathrm{~h}^{-}}$ CuPaz film drop-cast from solution in the benzene-DMAP system (20:1, $\mathrm{v} / \mathrm{v})$.

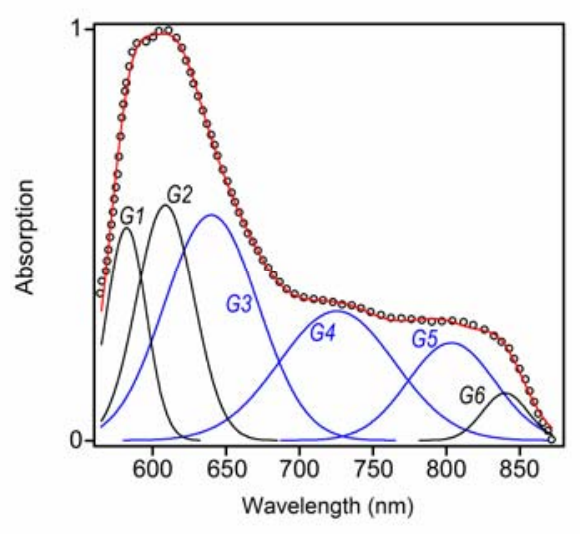

Figure S21. Gaussian deconvolution of the red curve in Figure S20. Parameters of the G1-G6 peaks are given in Table $\mathbf{S 2}$.

Table S2. Gaussian components of the spectrum in Figure S21.

\begin{tabular}{ccccc}
\hline \hline Peak & $\boldsymbol{\lambda}_{\boldsymbol{0}}$ & $\boldsymbol{\lambda}$ & $\boldsymbol{A}$ & $\boldsymbol{S}$ \\
\hline$G 1$ & 580 & 582 & 0.52 & 12.3 \\
\hline$G 2$ & 610 & 608 & 0.57 & 19.7 \\
\hline$G 3$ & 640 & 640 & 0.55 & 30.8 \\
\hline$G 4$ & 725 & 726 & 0.31 & 21.7 \\
\hline$G 5$ & 805 & 804 & 0.24 & 12.4 \\
\hline$G 6$ & 840 & 840 & 0.12 & 3.1 \\
\hline \hline
\end{tabular}

Abbreviations: $\lambda_{0}$ and $\lambda$, input and output centers, nm; $A$, height, rel. units; $S$, area, $\%$. 
Section B. Other films.

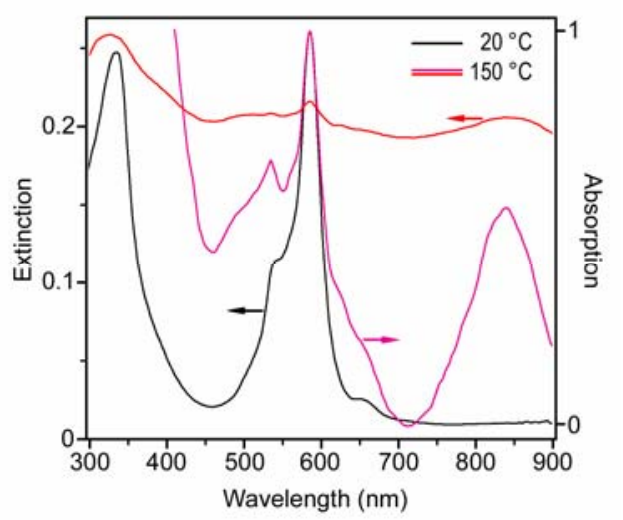

Figure S22. Optical spectra of the LS film of mix-CuPaz (30 layers on quartz). The spectrum of the annealed film is shown both as-measured (red) and normalized (pink).

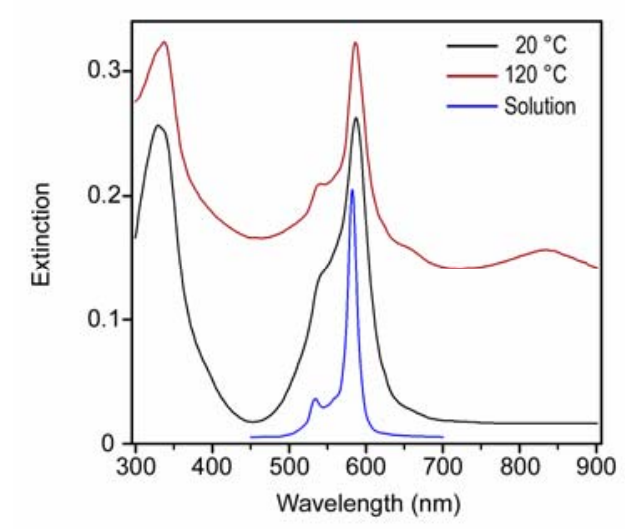

Figure S23. Optical spectra of the mix$\mathrm{CuPaz}$ film cast from benzene solution.

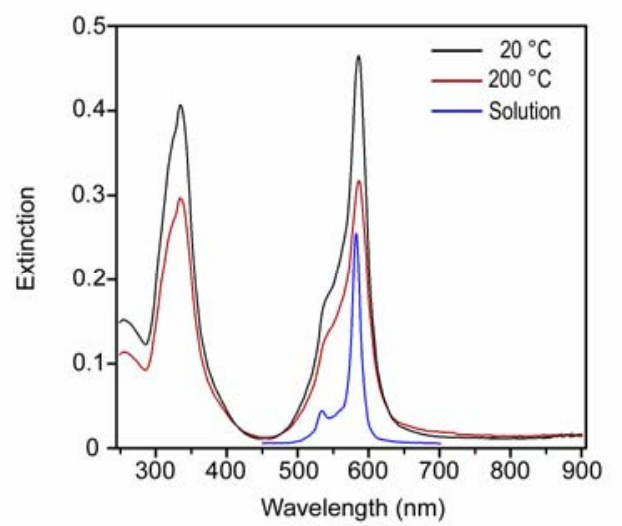

Figure S24. Optical spectra of the $\left(\mathrm{C}_{\mathrm{S}} / \mathrm{C}_{2 \mathrm{v}}\right)-\mathrm{CuPaz}$ film cast from benzene solution. Annealing time is $3 \mathrm{~h}$.

Notes: 1) The dye matter was partially sublimed off upon extra-severe annealing. 2) Relative light scattering is negligible both before and after heat treatment. 